Discussion Papers in

Economics and Econometrics

2000

This paper is available on our website http://www/soton.ac.uk/ econweb/dp/dp00.html 


\title{
Long Run Recursive VAR Models and QR Decompositions.
}

\author{
Mathias Hoffmann \\ Department of Economics \\ University of Southampton, U.K. \\ Highfield, Southampton SO17 1BJ \\ UNITED KINGDOM
}

Phone: ++44-(0)23-8059-2530 E-Mail: mh12@soton.ac.uk

July 26, 2000 


\begin{abstract}
Long-run recursive identification schemes are very popular in the structural VAR literature. This note suggests a two-step procedure based on QR decompositions as a solution algorithm for this type of identification problem. Our procedure will always deliver the exact solution and it is much easier to implement than a Newton-type iteration algorithm. It may therefore be very useful whenever quick and precise solutions of a long-run recursive schemes are required, e.g. in bootstrapping confidence intervals for impulse responses.
\end{abstract}

Keywords: Structural Vector autoregression, Long Run Recursive VAR, Choleski Decompositions, QR Decomposition, Economic Fluctuations, Cointegration 


\section{Introduction}

Starting with Blanchard and Quah (1989) and including further seminal papers such as Bayoumi and Eichengreen (19992 a,b), Taylor and Bayoumi (195), Clarida and Gali (1994) and Ahmed et al. (1993), longrun recursive identification schemes have proven to be of great practical relevance in the structural VAR literature.

Even though the usefulness of long-run identification restrictions has recently been questioned by some authors (see Faust and Leeper (1997), they remain very popular in applied work (e.g. Weber (1997), Rogers (1998)). This popularity is likely to derive from the fact that economic theory tends to make clear suggestions about the long-run whereas short run predictions are often less easy to come by.

The identification of structural shocks by means of a long-run identification scheme requires the solution of a non-linear system of equations. This is generally done by iterative, Newton-type algorithms. The present note suggests an alternative two-step solution procedure: First, some orthogonal decomposition of the reduced form variance-covariance matrix (e.g. a Choleski decomposition) is performed in order to orthogonalize the residuals. In a second step, the orthogonalized residuals are rotated by means of QR-decomposition of the long-run impact matrix. This procedure will always yield an exact solution whereas iterative techniques will almost certainly generate a rounding error. Finally, our procedure is easy to implement and may therefore be of considerable practical use in identifying long-run recursive vector autoregressive models.

The remainder of the paper is structured as follows: section 2 presents notation and a structural VAR model. Section 3 presents our two-step procedure. Section 4 extends the approach to the cointegrated case. Section 5 concludes.

\section{Identification of a structural VAR model}

We are considering models of the form

$$
\mathbf{A}(\mathbf{L}) \mathbf{X}_{t}=\varepsilon_{t}
$$

where $\mathbf{X}_{t}$ is a $(n \times 1)$ vector process, $\mathbf{A}(\mathbf{L})=\mathbf{I}-\sum \mathbf{A}_{i} \mathbf{L}^{i}$, the $\mathbf{A}_{i}$ are $(n \times n)$ matrices, $L$ is the lag operator $\mathbf{L}^{i} \mathbf{X}_{t}=\mathbf{X}_{t-i}$, and $\varepsilon_{t}$ is an $(n \times 1)$ i.i.d. disturbance term with mean zero and variance-covariance matrix $E\left(\varepsilon_{t} \varepsilon_{t}^{\prime}\right)=\Omega$. In the first part of the paper, we will assume that the matrix polynomial $\mathbf{A}(\mathbf{z})$ does not have roots on or outside the unit-circle, i.e. the process $\mathbf{X}(\mathbf{t})$ is stationary. We will later extend our results to the non-stationary, cointegrated case. 
By the Wold-theorem, $\mathbf{X}_{t}$ can be represented as a moving average of past disturbances:

$$
\mathbf{X}_{t}=\mathbf{C}(\mathbf{L}) \varepsilon_{t}
$$

where $\mathbf{C}(\mathbf{L})=\sum_{i=0}^{\infty} \mathbf{C}_{i} \mathbf{L}^{i}$ and the $\mathbf{C}_{i}$ are $(n \times n)$-matrices.

As is common in the structural VAR literature, the link between the reduced form and the structural model is established by the assumption that the reduced form residuals are just a linear combination of the structural disturbances:

$$
\varepsilon_{t}=\mathbf{S e}_{t}
$$

where $\mathbf{S}$ is a non-singular $(n \times n)$ matrix and the structural disturbances are contemporaneously uncorrelated, i.e. $\mathbf{E}\left(\mathbf{e}_{t} \mathbf{e}_{t}^{\prime}\right)=\mathbf{I}_{n}$.

It is then

$$
\mathbf{D}(\mathbf{L})=\mathbf{C}(\mathbf{L}) \mathbf{S}
$$

By (3), we get

$$
\boldsymbol{\Omega}=\mathbf{S E}\left(\mathbf{e}_{t} \mathbf{e}_{t}^{\prime}\right) \mathbf{S}^{\prime}=\mathbf{S S}^{\prime}
$$

Due to the symmetry of $\boldsymbol{\Omega}$, however, this gives only $n(n+1) / 2$ nonredundant restrictions on the matrix $\mathbf{S}$. Hence, another $n-n(n+1) / 2=$ $n(n-1) / 2$ restrictions have to be imposed on the model, for justidentification.

One way to get these missing restrictions, is to impose long-run recursiveness on the structural model.

Definition 1 The identification scheme is called long-run recursive iff

$$
\mathbf{D}(\mathbf{1})=\mathbf{C}(\mathbf{1}) \mathbf{S}
$$

is lower triangular.

\section{QR-decompositions and LR recursiveness}

The equation system implied by the identifying restrictions can be quite difficult to solve with standard numerical methods. Even in cases where convergence is easily achieved by the use of an appropriate method, iterative solution procedures will be quite inconvenient if the model is simulated, say for bootstrap estimation of confidence intervals of impulse responses. Our two step procedure always delivers an exact solution. It is quick and easy to implement with standard econometric packages. As such it is particularly well suited for the simulation of the structural model, bootstrap estimation of impulse responses and variance decompositions etc. 
The first step is to calculate any orthogonal decomposition of the covariance matrix $\Omega$. such that $\Omega=\mathbf{S}_{0} \mathbf{S}_{0}^{\prime}$. Now define

$$
\mathbf{e}_{0 t}=\mathbf{S}_{0}^{-1} \varepsilon_{t}
$$

and

$$
\mathbf{D}_{0}(\mathbf{1})=\mathbf{C}(\mathbf{1}) \mathbf{S}_{0}
$$

Obviously, $\mathbf{E}\left(\mathbf{e}_{o t} \mathbf{e}_{0 t}^{\prime}\right)=\mathbf{I}_{n}$. So, the orthogonalization of the residuals is already achieved. In particular, $\mathbf{S}_{0}$ could be chosen to be the Choleski factor of $\Omega$ as this procedure is part of almost any econometric package that allows VAR estimation. But in fact, any factorization such that $\mathbf{S}_{0} \mathbf{S}_{0}^{\prime}=\Omega$ will do for our purposes.

Before we give the second step, let us give the following result from linear algebra

Lemma $\mathbf{2}$ Let $\mathbf{Z}$ be a non-singular $(n \times n)$ matrix. Then there exists an upper triangular matrix $\mathbf{R}$ and an orthonormal matrix $\mathbf{Q}$ with $\mathbf{Q Q}^{\prime}=\mathbf{I}_{n}$ such that

$$
\mathrm{Z}=\mathrm{QR}
$$

Versions of this Lemma and a constructive proof can be found in many textbooks on numerical linear algebra, see for instance, Judd (1998). Procedures for the implementation of such a QR-decomposition are easily implemented or are even part of many standard packages. In MATLAB, for instance, the command line

$$
[\mathrm{Q}, \mathrm{R}]=\mathrm{qr}(\mathrm{Z})
$$

does the job, similar commands for OX and GAUSS are also available. Note that the QR decomposition can always be obtained by calculating $n-1$ Householder-transformations and that it is unique only up to the sign of the diagonal elements of $\mathbf{R}$.

The second step of the procedure is now as follows: let $\mathbf{Q}, \mathbf{R}$ define a QR decomposition of $\mathbf{D}_{0}(\mathbf{1})^{\prime}$. Then

$$
\mathbf{D}_{0}(\mathbf{1})=\mathbf{R}^{\prime} \mathbf{Q}^{\prime}=\mathbf{C}(\mathbf{1}) \mathbf{S}_{0}
$$

and accordingly because $\mathbf{Q}^{\prime} \mathbf{Q}=\mathbf{I}_{n}$

$$
\mathbf{C}(\mathbf{1}) \mathbf{S}_{0} \mathbf{Q}=\mathbf{R}^{\prime}
$$

Recall that $\mathbf{R}$ was defined to be upper triangular, hence, $\mathbf{R}^{\prime}$ is lower triangular. Note also that

$$
\mathbf{S}=\mathbf{S}_{0} \mathbf{Q}
$$


because

$$
\mathrm{SS}^{\prime}=\mathrm{S}_{0} \mathbf{Q Q}^{\prime} \mathrm{S}_{0}^{\prime}=\Omega
$$

and $\mathbf{C}(\mathbf{1}) \mathbf{S}$ is lower triangular. Consequently, $\mathbf{D}(\mathbf{1})=\mathbf{R}^{\prime}$ and the identification problem is solved. As the QR-decomposition is unique only up to the sign of the diagonal elements of $\mathbf{R}$, the columns of $\mathbf{R}$ and $\mathbf{Q}$ can now be appropriately normalized so that the long-run impulse response of the $i$-th variable to the $\vec{i}$-th shock has the desired sign.

\section{The cointegrated case}

In this section, we generalize our method to a cointegrated VAR. This gives rise to a three-stage procedure. The process $\mathbf{X}_{t}$ will now be $I(1)$ and cointegrated such that $\boldsymbol{\beta}^{\prime} \mathbf{X}_{t}$ is $I(0)$, for some $(n \times h)$-matrix $\boldsymbol{\beta}$. Let's consider the error correction representation:

$$
\boldsymbol{\Delta} \mathbf{X}_{t}=\sum_{i=1}^{k-1} \boldsymbol{\Gamma}_{i} \boldsymbol{\Delta} \mathbf{X}_{t-i}+\boldsymbol{\alpha} \boldsymbol{\beta}^{\prime} \mathbf{X}_{t-1}+\varepsilon_{t}
$$

where $\boldsymbol{\Gamma}_{i}=-\sum_{l=i+1}^{k-1} \mathbf{A}_{i}$ and $\boldsymbol{\alpha}, \boldsymbol{\beta}$ are full rank matrices of dimension $(n \times h)$ where $0 \leqq h \leqq n-1$ is the dimension of the cointegrating space. As is known from Stock and Watson (1988), in the cointegrated case the long-run impact matrix $\mathbf{C}(\mathbf{1})$ has rank $n-h$ and therefore, we will have to amend our notion of long-run recursiveness.

Definition 3 Let $\mathbf{S S}$ / $=\Omega$ and

$$
\mathbf{D}(\mathbf{1})=\mathbf{C}(\mathbf{1}) \mathbf{S}=\left[\begin{array}{ll}
\boldsymbol{\Psi} & \mathbf{0}_{n \times h}
\end{array}\right] \text { where } \boldsymbol{\Psi}=\left[\Psi_{R}^{\prime} \Psi_{H}^{\prime}\right]^{\prime}
$$

where $\Psi_{R}$ is $(n-h) \times(n-h)$ and $\Psi_{H}$ is $h \times(n-h)$. Then the identifying scheme represented by $\mathbf{S}$ is called long-run recursive iff $\Psi_{R}$ is lower triangular.

We will now propose a three-stage procedure to construct $\mathbf{S}$.

In the cointegrated case, the long-run impact matrix $\mathbf{C}(\mathbf{1})$ can be given the following representation (Johansen (1995)):

$$
\mathbf{C}(\mathbf{1})=\boldsymbol{\beta}_{\perp}\left(\alpha_{\perp} \Gamma(1) \beta_{\perp}\right)^{-1} \boldsymbol{\alpha}_{\perp}^{\prime}
$$

where $\boldsymbol{\alpha}_{\perp}, \boldsymbol{\beta}_{\perp}$ are the orthogonal complements of $\boldsymbol{\alpha}$ and $\boldsymbol{\beta}$. The matrix $\mathbf{C}(\mathbf{1})$ has rank $n-h$. Johansen (1995) has shown that the vector of permanent and transitory disturbances is given by

$$
\mathbf{u}_{t}=\left[\begin{array}{c}
\boldsymbol{\pi}_{t} \\
\boldsymbol{\tau}_{t}
\end{array}\right]=\left[\begin{array}{c}
\boldsymbol{\alpha}_{\perp}^{\prime} \\
\boldsymbol{\alpha}^{\prime} \boldsymbol{\Omega}^{-1}
\end{array}\right] \varepsilon_{t}=\mathbf{P}^{-1} \varepsilon_{t}
$$


The first step of our procedure is to generate the long-run impact matrix that is consistent with (13).It is easily verified that for any initial choice of $\boldsymbol{\alpha}_{\perp}$ and $\boldsymbol{\alpha}$, we have

$$
\mathbf{P}=\left[\boldsymbol{\Omega} \boldsymbol{\alpha}_{\perp}\left(\boldsymbol{\alpha}_{\perp}^{\prime} \boldsymbol{\Omega} \boldsymbol{\alpha}_{\perp}\right)^{-1}, \boldsymbol{\alpha}\left(\boldsymbol{\alpha}^{\prime} \boldsymbol{\Omega} \boldsymbol{\alpha}\right)^{-1}\right]
$$

Hence, it is computationally straightforward to get

$$
\mathbf{D}_{0}(\mathbf{1})=\mathbf{C}(\mathbf{1}) \mathbf{P}
$$

which simplifies to

$$
\mathbf{D}_{0}(\mathbf{1})=\left[\boldsymbol{\beta}_{\perp}\left(\boldsymbol{\alpha}_{\perp} \boldsymbol{\Gamma}(\mathbf{1}) \boldsymbol{\beta}_{\perp}\right)^{-1}, \mathbf{0}_{n \times h}\right]=\left[\boldsymbol{\Psi}_{0}, \mathbf{0}_{n \times h}\right]
$$

This completes stage one, i.e. the orthogonalization of permanent and transitory disturbances. However, permanent shocks among themselves and transitory shocks among themselves are not yet orthogonal. Rather, for arbitrarily chosen bases of $\operatorname{span}(\boldsymbol{\alpha})$ and $\operatorname{span}\left(\boldsymbol{\alpha}_{\perp}\right)$ after stage one we will have that

$$
\boldsymbol{\Sigma}=\mathbf{E}\left(\mathbf{u}_{t} \mathbf{u}_{t}^{\prime}\right)=\mathbf{P}^{-1} \boldsymbol{\Omega} \mathbf{P}^{-\mathbf{1} \prime}=\left[\begin{array}{cc}
\boldsymbol{\Omega}_{P} & \mathbf{0}_{n-h \times h} \\
\mathbf{0}_{h \times n-h} & \boldsymbol{\Omega}_{T}
\end{array}\right]=\left[\begin{array}{cc}
\boldsymbol{\alpha}_{\perp}^{\prime} \boldsymbol{\Omega} \boldsymbol{\alpha}_{\perp} & \mathbf{0}_{n-h \times h} \\
\mathbf{0}_{h \times n-h} & \boldsymbol{\alpha}^{\prime} \boldsymbol{\Omega} \boldsymbol{\alpha}
\end{array}\right]
$$

In stage two, we now choose bases of $\operatorname{span}\left(\boldsymbol{\alpha}_{\perp}\right)$ and $\operatorname{span}(\boldsymbol{\alpha}), \boldsymbol{\alpha}_{0 \perp}$ and $\boldsymbol{\alpha}_{0}$, such that $\boldsymbol{\alpha}_{0 \perp}^{\prime} \boldsymbol{\Omega} \boldsymbol{\alpha}_{0 \perp}=\mathbf{I}_{n-h}$ and $\boldsymbol{\alpha}_{0}^{\prime} \boldsymbol{\Omega} \boldsymbol{\alpha}_{0}=\mathbf{I}_{h}$. Very much as in the non-cointegrated case, any orthogonal decomposition of $\boldsymbol{\Sigma}$ will achieve this:

Again, let $\mathbf{S}_{0}$ define any decomposition of $\boldsymbol{\Sigma}$ such that $\boldsymbol{\Sigma}=\mathbf{S}_{0} \mathbf{S}_{0}^{\prime}$. Then $\mathbf{S}_{0}$ will be block diagonal and

$$
\boldsymbol{\Sigma}=\left[\begin{array}{cc}
\boldsymbol{\alpha}_{\perp}^{\prime} \boldsymbol{\Omega} \boldsymbol{\alpha}_{\perp} & \mathbf{0}_{n-h \times h} \\
\mathbf{0}_{h \times n-h} & \boldsymbol{\alpha}^{\prime} \boldsymbol{\Omega} \boldsymbol{\alpha}
\end{array}\right]=\mathbf{S}_{0} \mathbf{S}_{0}^{\prime}=\left[\begin{array}{cc}
\mathbf{S}_{0 \pi} \mathbf{S}_{0 \pi}^{\prime} & \mathbf{0}_{n-h \times h} \\
\mathbf{0}_{h \times n-h} & \mathbf{S}_{0 \tau} \mathbf{S}_{0 \tau}^{\prime}
\end{array}\right]
$$

Now choose $\boldsymbol{\alpha}_{0 \perp}=\boldsymbol{\alpha}_{\perp} \mathbf{S}_{0 \pi}^{-1^{\prime}}$ and $\boldsymbol{\alpha}_{0}=\boldsymbol{\alpha} \mathbf{S}_{0 \tau}^{-1 \prime}$ to complete the desired orthogonalization.

The second stage long-run impact matrix is then given by

$$
\mathbf{D}_{1}(\mathbf{1})=\mathbf{C}(\mathbf{1}) \mathbf{P S}_{0}=\mathbf{D}_{0}(\mathbf{1}) \mathbf{S}_{0}=\left[\boldsymbol{\Psi}_{0} \mathbf{S}_{0 \pi}, \mathbf{0}_{n \times(n-h)}\right]=\left[\boldsymbol{\Psi}_{1}, \mathbf{0}_{n \times h}\right]
$$

For stage three, now consider the partition $\boldsymbol{\Psi}_{1}=\left[\boldsymbol{\Psi}_{1 U}^{\prime} \boldsymbol{\Psi}_{1 L}^{\prime}\right]^{\prime}$ suggested in the above definition. Note that $\mathbf{D}_{1}(\mathbf{1})$ has full rank and hence also $\boldsymbol{\Psi}_{1}$. Let $\mathbf{Q}_{P}$ and $\mathbf{R}_{P}$ define a $\mathrm{QR}$ decomposition of $\boldsymbol{\Psi}_{1 U}^{\prime}$. Then $\Psi_{1} \mathbf{Q}_{P}=\mathbf{R}_{P}^{\prime}$ is lower triangular and hence

$$
\mathbf{D}(\mathbf{1})=\left[\boldsymbol{\Psi}_{1}, \mathbf{0}_{n \times h}\right] \mathbf{Q}=\left[\begin{array}{cc}
\boldsymbol{\Psi}_{R} & \mathbf{0}_{(n-h) \times h} \\
\boldsymbol{\Psi}_{H} & \mathbf{0}_{h \times h}
\end{array}\right] \text { where } \mathbf{Q}=\left[\begin{array}{cc}
\mathbf{Q}_{P} & \mathbf{0}_{n-h \times h} \\
\mathbf{0}_{h \times n-h} & \mathbf{I}_{h}
\end{array}\right]
$$


Hence, $\Psi_{1} \mathbf{Q}_{P}=\boldsymbol{\Psi}$, the long-run recursive impact matrix is given by $\mathbf{D}(\mathbf{1})=\mathbf{C}(\mathbf{1}) \mathbf{P S}_{0} \mathbf{Q}=\mathbf{C}(\mathbf{1}) \mathbf{S}$ and the structural disturbances by

$$
\mathbf{e}_{t}=\mathbf{Q}^{\prime} \mathbf{S}_{0}^{-1} \mathbf{P}^{-1} \varepsilon_{t}=\mathbf{S}^{-1} \varepsilon_{t}
$$

\section{Conclusion}

In this note we have suggested a simple two-stage procedure based on QR decompositions that makes the numerical identification of long-run recursive VARs much easier. Our method offers the advantage of an exact solution whereas general Newton-type algorithms are likely to generate rounding errors. The method is particularly well suited whenever a large number of exact solutions of the model is required, e.g. in bootstrapping confidence intervals of impulse responses.

\section{References}

[1] Ahmed S., Ickes B.W. ,Wang, P. and Yoo, B.S. (1993), 'International Business Cycles', The American Economic Review, 83(3): 335-359.

[2] Bayoumi, Tamim and Taylor, Mark P., (1995), "Macroeconomic Shocks, the ERM and Tripolarity," The Review of Economics and Statistics, 321-331.

[3] Bayoumi, Tamin and Eichengreen, Barry, (1992a), 'Shocking Aspects of European Monetary Union,' CEPR Discussion-Paper no. 643.

[4] Bayoumi, Tamin and Eichengreen, Barry, (1992b), 'Macroeconomic Adjustment under Bretton Woods and the Post-Bretton-Woods Float: An Impulse Response Analysis,' CEPR Discussion Paper no. 729 .

[5] Beveridge, Stephen and Nelson, Charles R., "A New Approach to Decomposition of Economic Time Series into Permanent and Transitory Components with Particular Attention to Measurement of the 'Business Cycle"', Journal of Monetary Economics 7, 151-173.

[6] Blanchard, O. and Quah, D., (1989), 'The Dynamic Effects of Demand and Supply Disturbances', American Economic Review, 79: 655-73.

[7] Clarida, R. and Gali, J. (1994), 'Sources of Real Exchange Rate Fluctuations: How important are nominal shocks?', CarnegieRochester Conference Series on Public Policy, 41, 1-56.

[8] Faust, J. and Leeper, E. M. (1997), 'When Do Long-Run Identifying Restrictions Give Reliable Results?' Journal of Business and Economic Statistics, 15(3): 345-53. 
[9] Johansen, Søren, (1995), Likelihood-Based Inference in Cointegrated Vector Autoregressive Models, Oxford University Press.

[10] Judd, K. L. (1998), Numerical Methods in Economics, MIT Press.

[11] Rogers, J. (1999), 'Monetary Shocks and Real Exchange Rates', Journal of International Economics, 49, 269-88.

[12] Stock, James H. and Watson, Mark W., (1988), "Testing for Common Trends", Journal of the American Statistical Association 83, 1097-1107.

[13] Weber, A. (1997), 'Sources of Purchasing Power Disparities between the G3 Economies', Journal of Japanese and International Economies, 11, 548-583. 